\title{
CONVENTIONAL TERRESTRIAL SYSTEM BY VLBI: A KINEMATIC APPROACH
}

\author{
H. B. Papo and J. Saleh \\ Technion, Israel Institute of Technology \\ Technion City, Haifa 32000 \\ Israel
}

The paper examines the potentials of VLBI time delay observables for the establishment and maintenance of a Conventional Terrestrial System (CTS). The CTS is defined in 2-D by the standard epoch positions and velocities of a network of control points located on a spherical reference surface.

VLBI time delay observables are ideally suited for the establishment of a CTS due to their sensitivity to the rotational motion of the observing stations with respect to inertial space (CIS). The rotational motion of a VLBI station with respect to the CIS is partitioned into individual or regional motion with respect to a discrete terrestrial coordinate frame and into global motion of the terrestrial coordinate frame with respect to the CIS. The global motion is mathematically described by the models and parameters of general precession, nutation and diurnal spin. The unmodelled part is defined as polar motion and spin-rate variations. The above three models together with the unmodelled polar motion and spin-rate variation determine at a given epoch the orientation of the CTS coordinate frame with respect to the CIS.

A kinematic model of analysis of the CTS resembles the system of a fundamental star catalogue. Each CTS control point defines the CTS by its two spherical coordinates at the standard epoch $T_{0}$ and by the coordinate time rates. The only type of measurement considered in this paper is VLBI time delay, observed between points of the CTS. However, VLBI time delay measurements alone are inadequate for defining completely the CTS in 2-D at a given epoch or for monitoring its rotational motion. There is a need for additional data sets and models (derived from non-VLBI sources) that would corvey a conventional datum to the CTS. The method of analysis that incorporates these data sets should be designed so as to preclude any distortion of the measurements.

The datum of a kinematic network becomes a problem when the observational material used for estimating coordinates and velocities of the individual points is deficient in content. The datum problem encountered in most large geodetic projects is solved by introducing additional and subjective information - the datum basis. It has the form of a set of point-coordinates, velocities or other relevant parameters with a common property of being somewhat less consistent as compared to the "inadequate" but objective observations. The subjective datum content of the basis is enforced without disturbing the consistency of the observations, through the application of free net constraints. The resulting solution has two desirable properties:

- the coordinate frame defined by the datum basis is maintained, i.e., the esti- 
mated coordinates define an identical coordinate frame; and

- the corrections to the measurements are independent of the specific datum basis. They reflect only the measurement noise.

The CTS treated as a kinematic entity needs two datum bases:

- the first datum basis (a set of point coordinates) defines the CTS frame at the standard epoch;

- the second basis consists of a set of velocities that refers to the same datum points.

An obvious choice for the first datum basis is the BIH coordinates of a selected number of coordinated observatories. Such a choice would insure a smooth transition from the present BIH/CIO terrestrial system to the CTS. For the second datum basis we recommend a set of point velocities derived from the parameters of a plate tectonics model. The advantages of adopting the second basis are:

- reduced sensitivity to CTS point distribution;

- minimization of the motion of the asthenosphere as represented by a network of hot spots;

- velocities are minimized using weights that reflect the quality of the plate tectonics model parameters.

We consider an extended period of time (years) during which all CTS control stations participate in VLBI measurements. A small subset of three to four stations observes continuously while the remaining majority observes from time to time. The parameters that can be estimated are grouped as follows: (1) earth orientation parameters; (2) CTS station standard epoch coordinates; (3) CTS station velocities; (4) radio source CIS coordinates; and (5) station clock error model parameters.

A simulation study in which four CTS stations participated in VLBI measurements over five years for a total of only five days resulted in the following standard deviations:

\begin{tabular}{lcllr}
\hline $\begin{array}{l}\text { Date of } \\
\text { observation }\end{array}$ & $\begin{array}{c}\text { Accumulated } \\
\text { days }\end{array}$ & $\begin{array}{l}\text { Earth orie. } \\
\text { (cm) }\end{array}$ & $\begin{array}{l}\text { CTS pos. } \\
\text { (cm) }\end{array}$ & $\begin{array}{l}\text { CTS velo. } \\
\text { (cm/year) }\end{array}$ \\
\hline $1-1-81$ & 1 & $8-10$ & $5-23$ & - \\
$1-6-81$ & 2 & $6-9$ & $4-17$ & $100 .-300$. \\
$1-1-82$ & 3 & $6-8$ & $3-15$ & $1.7-3.5$ \\
$1-1-83$ & 4 & $5-8$ & $1-14$ & $.7-$ \\
$1-1-86$ & 5 & $5-7$ & $1-12$ & $.3-$ \\
\hline
\end{tabular}

We believe that the idea of treating the Conventional Terrestrial System as a kinematic entity should be adopted by the scientific community as a standard method of analysis. Although crude and unsophisticated in technical detail, our studies demonstrate the high effectiveness of VLBI for the establishment and maintenance of a kinematic CTS. The main characteristics of the proposed kinematical approach to CTS analysis are:

- point velocities are estimated directly without having to resort to multi-stage adjustment techniques with the inevitable but dubious assumptions on error propagation;

- CTS datum determination and maintenance are explicitly associated with discrete data sets;

- the adjustment procedure is sequential in the true sense. CTS parameters are updated continuously at the rate of the incoming data. 